\title{
Analysis of surgical management and outcome of Blunt abdominal trauma
}

\author{
Kane V. ${ }^{1}$, Dhandore C.D. ${ }^{2}$ \\ ${ }^{1}$ Dr. Varsha Kane, ${ }^{2}$ Dr. Chetan D. Dhandore, both authors are Associate Professor, Department of General Surgery, \\ Ashwini Rural Medical College, Hospital \& Research Centre, Kumbhari, Solapur, Maharastra, India. \\ Correspondence Author: Dr. Chetan D. Dhandore, Associate Professor, Department of General Surgery, Ashwini Rural \\ Medical College, Hospital \& Research Centre, Kumbhari, Solapur, Maharastra, India. E-mail: \\ drchetandhandore@gmail.com
}

\begin{abstract}
Background: Trauma, in particular, blunt abdominal trauma, associated with vehicular accidents and railway accident, has become major cause of morbidity and mortality for working population in developing and industrial nations of world. Hence the present study was undertaken to assess the surgical management and outcome of Blunt abdominal trauma. Method: 49 cases of blunt abdominal trauma studied during study period in a tertiary care hospital. On admission a relevant history with nature of accident, time of occurrence and injuries sustained were noted. A detailed examination of the patient was done, which included general examination with appropriate recording of pulse, blood pressure, and respiratory rate at time of admission, and systemic examination of chest abdomen and central nervous system. Details of external, skeletal and other associated injuries were noted. Presence or absence of hematuria was also recorded. Result: Predominantly younger population (21-30) is affected by trauma with male (10-1) preponderance. Vehicular accident was the commonest cause (48.4\%). Blind abdominal tap was 53\% sensitive for hemoperitoneum while USG $90 \%$. CT was almost $100 \%$ sensitive for abdominal injuries. Spleen $(53.08 \%)$ and liver $(22.44 \%)$ were the common organs injured. Associated injuries were present in $42.85 \%$ cases. Mortality was higher in those operated beyond one hour. Conclusion: Prevention and measures to decrease morbidity and mortality from abdominal trauma is essential to avoid loss of productive years of life. Trauma registry with documentation of care delivered, assessment of outcome and implementation of necessary changes would help in providing better care.
\end{abstract}

Keywords: Blunt abdominal trauma, Vehicle accident, Liver injury, Spleen

\section{Introduction}

Trauma is the third most common cause of death overall leading to disability in the first four decades of life. Trauma is characterized by a structural alteration or physiological imbalance that results when energy is imparted during interaction with physical or chemical agents. Trauma, in particular, blunt abdominal trauma, associated with vehicular accidents and railway accident, has become major cause of morbidity and mortality for working population in developing and industrial nations of world.

It represents a significant source of economic resource diversion in all modern societies, especially those that offer a package of health care benefits to their citizens. India had fourth highest rate of road accidents in the world, the first being USA.

Manuscript Received: $6^{\text {th }}$ May 2019 Reviewed: $16^{\text {th }}$ May 2019

Author Corrected: $24^{\text {th }}$ May 2019

Accepted for Publication: 28 ${ }^{\text {th }}$ May 2019
Trauma has no anatomical barriers. More than one region may be affected simultaneously, so patient should be treated as a whole. Abdomen is the third most common injured region and blunt abdominal trauma is more common than penetrating abdominal trauma [1]. The greatest difficulty in management of blunt abdominal trauma is the diagnosis, as it is masked by other injuries like head injury, chest injury and fractures. Intestinal disruptions can be due to a variety of types of blunt trauma, with automobile being the most common aetiologic agent [2,3]. Thus blunt abdominal trauma is a condition in which high index of suspicion is required alongwith active investigations to diagnose intraperitoneal injuries. The primary goal in the treatment of severe abdominal injury is to preserve life. The management is divided into four sequential phases, resuscitation, evaluation, initial management and definitive treatment. 
Objectives: To assess the surgical management and outcome of Blunt abdominal trauma

\section{Material and Methods}

Type of study: Prospective study

Sample collection: Blunt abdominal trauma cases selected during study period

\section{Selection criteria}

Inclusion criteria: Patient with blunt abdominal trauma case.

Exclusion criteria: Patient not having blunt abdominal trauma case

Surgical Procedure: 49 cases of blunt abdominal trauma studied during study period in a tertiary care hospital. On admission a relevant history with nature of accident, time of occurrence and injuries sustained were noted.

A detailed examination of the patient was done, which included general examination with appropriate recording of pulse, blood pressure, and respiratory rate at time of admission, and systemic examination of chest abdomen and central nervous system. Details of external, skeletal and other associated injuries were noted. Presence or absence of hematuria was also recorded.

Airway was cleared, after clearing of airway and maintenance of breathing and circulation was done by positioning, oxygen, intubation, ventilation, cervical spine immobilization, venous cannula or cut down. A preoperative sample of blood for hemoglobin, packed cell volume, serum electrolytes, sugar, blood for grouping and crossmatching was sent. Fluid resuscitation with Ringer Lactate, colloids, blood was started. Urinary catheterization done (except in cases of suspected urethral injuries) and presence or absence of hematuria noted.

Four quadrant abdominal tap was done to look for haemoperitoneum or faecal contamination in cases of suspected blunt trauma to the abdomen. It was also done in all unconscious patients where exact nature of accident could not be ascertained, especially in patients presenting with signs of shock.

X-ray of the abdomen, chest with both domes of diaphragm and other injured parts were taken with a portable $\mathrm{X}$ - ray. An ultrasound examination of the

\section{Original Research Article}

abdomen was carried out in cases, which were stable hemodynamically if. a) Organ injury suspected i.e. Pallor, reversed shock, local signs despite a negative abdominal tap. B) In patients with no local signs of trauma where intra - abdominal injury could not be ruled out

An emergency intravenous pyelogram was obtained in patients with suspected genitourinary injuries where presence of hematuria, loin swelling or perinephric or massive retroperitoneal hematoma on abdominal sonography was obvious.

C. T. scan to evaluate stable patients with suspected or U. S. G. detected injuries to grade and plan conservative line of management. Laparotomy was done as early as possible after a written valid and informed consent.

The indications for laprotomy were:

1. Positive abdominal tap.

2. Local abdominal signs of peritonitis such as presence of guarding and rigidity.

3.USG detected organ injury which could not be conserved.

4. Other radiological evidence of intra abdominal injury such as free gas under diaphragm.

A midline approach was used as a standard protocol in all cases. The findings it laparotomy were noted as-

1.Amount of hemoperitoneum or pus and fecal and biliary contamination.

2. Organ injured and the site and extent of injury.

3. The state of viscera and any other incidental findings.

The procedures done varied as per the organ's injured. A monolayer interrupted closure with monofilament was used in all cases after peritoneal wash. Drains were left in peritoneal cavity in all cases. Post operatively, patients were managed on IV fluids, blood transfusions, broad-spectrum antibiotics and analgesics.

Complications, if any were recorded and dealt with accordingly. Patients on recovery were discharged and followed on OPD basis. Autopsy was conducted in all expired cases to ascertain the exact cause of death.

Statistical methods: The collected data entered in Microsoft excel. Tables and graphs were generated by using Microsoft excel. Descriptive statistics such as mean, SD and percentage was used to present the data. 


\section{Observations}

Table-1: Basic Characteristics.

\begin{tabular}{|c|c|c|}
\hline Characteristics & No. of patients & \% \\
\hline Age Groups (Years) & & 20.6 \\
\hline $0-20$ & 15 & 44.8 \\
\hline $21-30$ & 22 & 12.24 \\
\hline $31-40$ & 6 & 10.20 \\
\hline $41-50$ & 5 & 2.04 \\
\hline 51 and above & 1 & 93.87 \\
\hline Sex & & 6.12 \\
\hline Male & 46 & \\
\hline Female & 3 & \\
\hline
\end{tabular}

Blunt abdominal trauma was seen at all ages, predominantly in the prime of life between 21-30 years (44\%). Incidence in paediatric age group is less as they are protected while elderly is too sedentary to be prone for injuries.

The sex distribution has a male preponderance and male: female ratio is 15.3:1. The reason behind the male preponderance is due to their high working population.

Table-2: Distribution of Injury Related Parameters

\begin{tabular}{|c|c|c|c|c|}
\hline Parameters & No. of Patients & $\%$ & Mortality & $\%$ \\
\hline \multicolumn{5}{|l|}{ Mode of Injury } \\
\hline Vehicular accidents & 20 & 40.81 & 1 & 5.00 \\
\hline Railway accidents & 14 & 28.57 & 6 & 42.85 \\
\hline Fall & 10 & 20.48 & 3 & 27.27 \\
\hline Assaults & 5 & 10.20 & 0 & 0 \\
\hline \multicolumn{5}{|l|}{ Associated injuries } \\
\hline$>2$ injuries & 7 & 14.28 & 2 & 28.6 \\
\hline Head injury & 10 & 20.48 & 9 & 90 \\
\hline Chest injuries & 5 & 10.70 & 1 & 20.0 \\
\hline Pelvic injuries & 6 & 12.24 & 1 & 16.7 \\
\hline Spine fracture & 0 & 0 & 0 & 0 \\
\hline Extremity fracture & 8 & 16.32 & 3 & 37.5 \\
\hline Injury present & 21 & 42.85 & 10 & 47.6 \\
\hline \multicolumn{5}{|l|}{ Management of injuries } \\
\hline Conservative & 13 & 26.53 & 1 & 7.69 \\
\hline Operative & 36 & 73.46 & 9 & 25 \\
\hline \multicolumn{5}{|l|}{ Time interval } \\
\hline$<$ one hour & 5 & 10.20 & 0 & 0 \\
\hline 1-24 hours & 41 & 83.45 & 9 & 21.95 \\
\hline$>24$ hours & 3 & 6.12 & 1 & 33.33 \\
\hline
\end{tabular}

Vehicular accidents have been the major cause of trauma. In our study, they contribute $40.81 \%$ followed by railway accident $28.57 \%$.

Chest injuries included fracture ribs, pneumothorax and haemothorax. Only patients with significant head injury detected on CT scan were taken into consideration. Limb fractures, pelvic fractures and significant soft tissue injuries were classified as other injuries. 
9 out of $10(90 \%)$ patients who died had associated with head injuries. Pelvic fracture was seen in 1 out of 10 patients. One patient with associated pelvic fracture died due to septicemia following jejuna perforation.

In conservative and operative management, mortality rate is 7.69 and $25 \%$ respectively. This is mainly due to associated injures.

The time interval between the events and definitive treatment showed that $6.12 \%$ of patients were taken up for surgery within one hour with mortality $0 \%$. The mortality rate was $21.95 \%$ in those who operated between $1-24$ hours and $33.33 \%$ in those who were operated after 24 hours.

Table-3: Organ Injury.

\begin{tabular}{|c|c|c|}
\hline Organ & Injury & \% \\
\hline Liver & 11 & 22.44 \\
\hline Spleen & 26 & 4.08 \\
\hline Kidney & 2 & 4.08 \\
\hline Bladder & 2 & 2.04 \\
\hline Pancreas & 1 & 4.08 \\
\hline Stomach \& Duodenum & 2 & 12.24 \\
\hline
\end{tabular}

Spleen was the commonest organ injured with 55.06 of patients. Liver was the next common organ injured being found in $22.44 \%$ of patients, with mortality of $12.5 \%$. This was followed by intestine and mesentry, then kidney and bladder injuries.

Table-4: Sensitivity of Investigations.

\begin{tabular}{|c|c|c|c|c|}
\hline Tap & Total Cases & + VE & -VE & Sensitivity \\
\hline Blind Abd. Tap & 49 & 26 & 23 & 53 \\
\hline USG guided tap & 10 & 9 & 1 & 90 \\
\hline
\end{tabular}

26 patients $(53 \%)$ were detected on positive abdominal tap while 9 out of 10 patients $(90 \%)$ were detected on USG guided abdominal tap while 1 patient $(10 \%)$ had negative abdominal tap. Few initial negative Four QTAP turned positive on subsequent taps after fluid resuscitation.

Gas under diaphragm was seen on radiology suggestive of bowel perforation in 4 cases. One ileal perforation could not be diagnosed on initial radiograph but USG guided tap was bilious.

Table-5: Relation between mode of injury and organ.

\begin{tabular}{|c|c|c|c|c|c|c|}
\hline Organ & Vehicular & Fall & Assault & Railway & Total & \% \\
\hline Spleen & 10 & 5 & 1 & 10 & 26 & 53.1 \\
\hline Liver & 5 & 2 & 2 & 2 & 9 & 16.32 \\
\hline Small Intestine and Mesentry & 3 & 1 & 1 & 1 & 6 & 12.24 \\
\hline Kidney and Bladder & 2 & 0 & 2 & 0 & 4 & 8.16 \\
\hline Duodenum and stomach & 0 & 1 & 1 & 0 & 2 & 4.08 \\
\hline Pancreas & 0 & 1 & 0 & 0 & 1 & 2.04 \\
\hline Vascular injury & 0 & 0 & 0 & 1 & 1 & 2.04 \\
\hline Total & $\mathbf{2 0}$ & $\mathbf{1 1}$ & $\mathbf{5}$ & $\mathbf{1 4}$ & & \\
\hline
\end{tabular}

Spleen was most commonly injured organ followed by liver. Vehicular accident was the commonest mode of injury. 
Original Research Article

Table-6: Post Operative Complication.

\begin{tabular}{|l|c|c|c|c|}
\hline Complications & No. & \% & Deaths & Mortality \\
\hline Chest infection & 6 & 16.66 & 0 & 0 \\
\hline Septicaemia / ARDS & 1 & 2.77 & 1 & $100 \%$ \\
\hline Neurological deterioration due to head injury & 9 & 22.22 & 9 & $100 \%$ \\
\hline Wound injury & 3 & 8.33 & 0 & 0 \\
\hline Burst abdomen & 1 & 2.77 & 0 & 0 \\
\hline
\end{tabular}

9 patients died due to head injury and 1 patient died due to ARDS and speticiemia.In conserved patients 1 patient died due to head injury.

\section{Discussion}

In the present study, age incidence varied from 5 years to 80 years and the highest incidence $44.8 \%$ was between 21-30 years compared to $32 \%$ in study by Gurguis [4]. This can be attributed to heavy vehicular traffic over crowded suburban railways, inter gang rivalry where young adults are mainly involved. Other authors also observed involvement of similar age groups in their study $[5,6]$.

Males were 15 times more injured (93.8\% vs $6.12 \%$ ) as in study. Mortality in the ratio of 10:0. In the present study, vehicular accidents (40.80\%) were the commonest mode of injury of blunt abdominal trauma compared to $80 \%$ [6]. Railway accident caused $42.85 \%$ of deaths, while due to vehicular accidents $5.0 \%$ and due to fall- $27.27 \%$. Whereas, in another study, it was reported that road traffic accidents $(61 \%)$ followed by railway accidents (28\%), fall (26\%), assault 14.28\% [7]. There was no case of seat belt injury in present study.

Injury to admission interval was less than one hour in only $10.20 \%$ in contrast to Eastman, goal for prehospital time of less than 30 minutes in urban environment [8]. But in our country, the concept of field resuscitation by paramedical squad is unknown and helicopter ambulance is perhaps for future. It is difficult also in India due to financial constraint.

Hence according to the concept of "Golden Hour" the first hour after trauma is lost in most of the victims as $82 \%$ of our population lives in the rural areas [9]. The time elapsed before definitive treatment is vital to the outcome as seen by trimodal distribution of death in trauma patients.

The time interval between admission and surgery was less than 1 hour in $6.12 \%$ cases and mortality was $0 \%$ less compared to delay in cases. $33.33 \%$ in those delayed beyond 24 hours for investigations. This emphasizes the importance of golden hour and silver day as in other studies by, Obert Blow [9], Gupta S. Talwar [10], Oreskovithch MR [11], and Nast Kolb [12]. Delay in repair of small bowel injury leads to increase in the morbidity and mortality. Similar kind of explanation had been given in ileal perforation [13].

Abdominal tenderness and guarding are common predictors. Tachycardia and hypotension are associated with high mortality. This is similar to findings of study by Clark J. R [14].

Associated Injury: By distracting attention from abdominal injury, there is increases mortality and morbidity directly and indirectly. 1 patient out of 10 who died had associated injures. 9 had head injuries associated with pelvic fracture and septicemia.

$18.18 \%$ liver injuries and $7.69 \%$ splenic injuries had associated rib fracture emphasizing the risk of abdominal organ injury in cases with rib fracture [15].

Management: Out of 49, 36 underwent surgery and 13 patient managed conservatively. Early resuscitation, better monitoring and routine CT abdomen would identify lesser grade injuries and prompt conservative management [16].

Investigations: Abdominal tap was found to be quick and reliable methods in the diagnosis of blunt abdominal injuries. Blind abdominal tap was positive in $53 \%$ and USG guided tap positive in $90 \%$ where abdominal tap was negative as compared to $76 \%$ in Abu Zidan [17], 84\% in Brown MA [18]. This shows the importance of portable sonography facility in trauma ward, FAST (Focused Assessment for Sonographic evaluation of Trauma patient) training. USG is false negative in injuries to retroperitoneum, bowel and solid organs without hemoperitoneum [19]. 
DPL was not done and CT done for USG positive and clinically suspected USG negative patients in the present study and findings were similar to study by Brown MA [18]. In 1 case of blunt abdominal trauma, it is found that USG normal but CT suggestive of liver laceration.

Sensitivity of CT was $100 \%$. It is needed for lesions of hollow viscus and solid organs as usefulness index of USG is 0.0069 as per Abu Zidan [17]. It is more sensitivity than USG to detect occult injuries when pelvic ring fractures are present [19]. Few initial negative Four quadrant Tap turned positive after fluid resuscitation directed to a target SBP of 90-100. This emphasizes the phenomena of increased blood loss with overzealous infusions and gives a thought for permissive hypotension and dry management of injuries till definitive repair of control of source of bleeding.

Complications: Analysis of the cases that expired shows neurological deterioration associated with 9 cases, infection, septicemia, multiple organ failure in other cases.

A case of jejuna perforation, which was detected on day 5 after injury and sutured, leaked after 5 days. Reexploration and resection anastomosis was done. Patient died of septicemia, ARDS. This case emphasizes the importance of DPL and CT scan in early detection of bowel injuries. Increased accessibility to CT scans facility both geographically and financially with intensive care management should improve early detection of neurological deterioration, bowel injury and prevent the mortality.

\section{Conclusion}

We can conclude that a possibility of blunt abdominal trauma should be kept in mind in all cases of polytrauma and vehicular accidents, even when local signs are absent. Thus prevention and measures to decrease morbidity and mortality from abdominal trauma is essential to avoid loss of productive years of life. Strict enforcement of traffic rules and regulations with better transport system, education and safety measures help in prevention.

Trauma registry with documentation of care delivered, assessment of outcome and implementation of necessary changes would help in providing better care.

What this Study adds to existing knowledge? Trauma registry with documentation of care delivered, assessment of outcome and implementation of

\section{Original Research Article}

necessary changes would help in providing better care, which makes a meaningful adding in existing literature by conducting our study.

Conflict of interest: None declared.

Funding: Nil, Permission from IRB: Yes

\section{References}

1. Hoyt David B, Coimbra R, Winchell RJ. Management of acute trauma. Sabiston Textbook of Surgery the biological basis of modem surgical practice, 16th edition, edt. by Town Sen Courtney,-WB Saunders Company. 2001:311-41.

2. Shuck JM, Lowe RJ. Intestinal disruption due to blunt abdominal trauma. Am J Surg. 1978 Dec;136(6): 668-73.

3. Munns J, Richardson M, Hewett P. A review of intestinal injury from blunt abdominal trauma. Aust N Z J Surg. 1995 Dec;65(12):857-60.

4. Guirguis EM, Hong C, Liu D, et al. Trauma outcome analysis of two Canadian centres using the TRISS method. J Trauma. 1990 Apr;30(4):426-9.

5. Rhodes M, Aronson J, Moerkirk G, et al. Quality of life after the trauma center. J Trauma. 1988 Jul;28 (7): 931-8.

6. Kreis JD, Fine EG, Gomez GA, Eckes JE, Whitwell EN, Byers PM. A prospective evaluation of field categorization of trauma patients. The Journal of trauma. 1988 Jul;28(7):995-1000.

7. Sayers RD, Bewes PC, Porter KM. Emergency laparotomy for abdominal trauma. Injury. 1992; 23(8):537-41.

8. Eastman A. The formation, isolation and characterrization of DNA adducts produced by anticancer platinum complexes. Pharmacol Ther. 1987; 34 (2): 155-66.

9. Blow O, Magliore L, Claridge JA, et al. The golden hour and the silver day: detection and correction of occult hypoperfusion within 24 hours improves outcome from major trauma. J Trauma. 1999 Nov;47 (5): 964-9.

10. Gupta S, Talwar S, Sharma RK, et al. Blunt trauma abdomen: a study of 63 cases. Indian J Med Sci. 1996 Aug; 50 (8):272-6. 


\section{Original Research Article}

11. Oreskovithch MR, Carrico E. Management of acutely injured patients. Textbook of surgery. Vol 1. Philadelphia lgakustrion / Saunders. 1986; 17: 294 - 299

12. Nast-Kolb D, Waydhas C, Kastl S, et al. [The role of an abdominal injury in follow-up of polytrauma patients]. Chirurg. 1993 Jul;64(7):552-9.

13. Fakhry SM, Brownstein $M$, Watts DD, et al. Relatively short diagnostic delays ( $<8$ hours) produce morbidity and mortality in blunt small bowel injury: an analysis of time to operative intervention in 198 patients from a multicenter experience. J Trauma. 2000 Mar;48 (3):408-14; discussion 414-5.

14. Clarke JR, Trooskin SZ, Doshi PJ, et al. Time to laparotomy for intra-abdominal bleeding from trauma does affect survival for delays up to 90 minutes. J Trauma. 2002 Mar;52(3):420-5.

15. Shweiki E, Klena J, Wood GC, et al. Assessing the true risk of abdominal solid organ injury in hospitalized rib fracture patients. J Trauma. 2001 Apr;50(4):684-8.
16. Moore EE, Malangoni MA, Cogbill TH, et al. Organ injury scaling. IV: Thoracic vascular, lung, cardiac, and diaphragm. J Trauma. 1994 Mar; 36 (3): 299300.

17. Abu-Zidan FM, Sheikh M, Jadallah F, et al. Blunt abdominal trauma: comparison of ultrasonography and computed tomography in a district general hospital. Australas Radiol. 1999 Nov;43(4):440-3.

18. Brown MA, Casola G, Sirlin CB, et al. Blunt abdominal trauma: screening us in 2,693 patients. Radiology. 2001 Feb; 218 (2):352-8. DOI: 10.1148/ radiology. 218.2.r01fe42352

19. Ballard RB, Rozycki GS, Newman PG, et al. An algorithm to reduce the incidence of false-negative FAST examinations in patients at high risk for occult injury. Focused Assessment for the Sonographic Examination of the Trauma patient. J Am Coll Surg. 1999 Aug;189(2):145-50; discussion 150-1.

\section{How to cite this article?}

Kane V, Dhandore C.D. Analysis of surgical management and outcome of Blunt abdominal trauma. Surgical Update: Int J surg Orthopedics. 2019;5(2):80-86.doi:10.17511/ijoso.2019.i02.03. 